\title{
Existence of Generalized Nash Equilibrium in $n$-Person Noncooperative Games under Incomplete Preference
}

\author{
Xingchang Li $\mathbb{D}^{1,2}$ \\ ${ }^{1}$ School of Mathematics and Statistics, Changshu Institute of Technology, Suzhou 215500, China \\ ${ }^{2}$ Institute of Business \& Economic Research, Harbin University of Commerce, Harbin 150028, China \\ Correspondence should be addressed to Xingchang Li; lxctsq@163.com
}

Received 18 April 2018; Accepted 25 September 2018; Published 9 October 2018

Academic Editor: Xinguang Zhang

Copyright (c) 2018 Xingchang Li. This is an open access article distributed under the Creative Commons Attribution License, which permits unrestricted use, distribution, and reproduction in any medium, provided the original work is properly cited.

\begin{abstract}
To prove the existence of Nash equilibrium by traditional ways, a common condition that the preference of players must be complete has to be considered. This paper presents a new method to improve it. Based on the incomplete preference corresponding to equivalence class set being a partial order set, we translate the incomplete preference problems into the partial order problems. Using the famous Zorn lemma, we get the existence theorems of fixed point for noncontinuous operators in incomplete preference sets. These new fixed point theorems provide a new way to break through the limitation. Finally, the existence of generalized Nash equilibrium is strictly proved in the $n$-person noncooperative games under incomplete preference.
\end{abstract}

\section{Introduction and Preliminaries}

As a kind of strategy combinations, Nash equilibrium is closely bound up with many important mathematical problems, and many problems in economy and engineering technology can also be described as a Nash equilibrium problem. Recently, the existence of the Nash equilibrium of noncooperative games has been studied [1-4]. In [1], the existence of uncertainty for generalized Nash equilibrium is proved by introducing the uncertainty to study generalized games. Using maximization theorem, the author presented the existence of Nash equilibrium in generalized games, and in these results, the strategy set is noncompact and has infinite players [2]. In [3] the existence theorem of generalized Nash equilibrium in games is given where strategy space has abstract convex structure. Assuming strategy set is a $H$ space, the equilibrium existence theorems have been given in [4]. In the above studies, either the partial order relation on policy sets is required to satisfy, or every total order subset in policy sets must have an upper bound or certain convexity condition.

For a long time, the preference of rational decisionmakers on management and economics should satisfy the completeness. But in practice, they show the indecision on many major issues. Since the preference without completeness is a kind of more general order structure, it can make preference relation and the partial order relation unified completely. The research of preference without completeness is started from the von Neumann and Morgenstern in [5]. Aumman and Bewley have made the classic study in $[6,7]$; Schmeidler has studied the existence of economic equilibrium with infinite number of institutions under incomplete preference in [8].

In noncooperative games, the policy set composed of player's selection strategies is a set which cannot meet the needs of completeness. If preference does not meet the completeness, Pareto optimality is meaningless and the traditional method of partial order will lose effectiveness without the antisymmetry axiom inevitably. Therefore, it is consistent with the realistic decision-making environment to study the existence of generalized Nash equilibrium of noncooperative game, but the study of this part has seldom been seen in the past literature research.

In this article, based on the equivalence class set which corresponds to the elements of incomplete preference set being a partial order set, the problem under incomplete preference is translated into the problem with partial order. This method overcomes the difficulties which are brought about by the elements in the set without the completeness. 
Using the famous Zorn lemma, we get the existence theorems of fixed point for noncontinuous operators in incomplete preference sets. The fixed point theorems provide a new way for breaking through the limitations. The existence of generalized Nash equilibrium is strictly proven in the $n$-person noncooperative games under incomplete preference.

Here some concepts and theorems are given, which are related to incomplete preference.

Let $E$ be a nonempty set. An ordering relation $\preceq$ on $E$ may satisfy the following axiom:

Reflexive: $x \preceq x$, for any $x \in E$;

Symmetry: If $x \preceq y$ and $y \preceq x$, then $x=y$, for any $x, y \in E$; Transitive: If $x \preceq y$ and $y \preceq z$, then $x \preceq z$,for any $x, y, z \in E$; Complete: If $x \preceq y, y \preceq x$, for any $x, y \in E$, there is at least one inequality to be established.

Definition 1 (see [9]). Let $E$ be a nonempty set. An order relation $\leq$ defined among certain elements of $E$ is said to be partial order if the order relation satisfies reflexive, transitivity, and antisymmetry axioms. Then $(E \leq)$ is called a poset.

Definition 2 (see [10]). Let $E$ be a nonempty set. An order relation defined among certain elements of $E$ is said to be incomplete preference order if the order relation satisfies reflexive and transitivity axioms, which is denoted by $\preceq$. If completeness axiom is still satisfied for incomplete preference order, the order relation is said to be preference order, which is still denoted by $\preceq$. Then $(E \preceq)$ is called an incomplete preference set.

Definition 3 (see [11]). Let $E$ be an incomplete preference set. For any $x, y \in E$, we say that $x, y$ are indifference, which is denoted by $x \sim y$, whenever both $x \preceq y$ and $y \preceq x$ hold.

Remark 4. $x \sim y \nRightarrow x=y$, but $x=y \Longrightarrow x \sim y$.

Remark 5. The indifference relation $\sim$ the equivalence relation.

Definition 6 (see [11]). Let $E$ be an incomplete preference set. If for any complete preference subset of $E$, there is denumerable set $\left\{x_{n}\right\} \subset M$ such that if $x \in M, x \neq \sup M$, there is $x_{n_{0}} \in\left\{x_{n}\right\}$ which satisfies $x \preceq x_{n_{0}}$, then $E$ is said to be pseudo separable in incomplete preference.

Let $E$ be an incomplete preference set, and $\Omega$ is a subset in $E$. The order relation $\leq$ in quotient set $\Omega / \sim$ is elicited by the incomplete preference relation $\preceq$ in $E$. Let $[x]=\{y \in \Omega \mid$ $x \sim y\}$, and $[x]$ is an equivalence class set in $\Omega$.

Definition 7 (see [11]). For any $[x],[y] \in \Omega / \sim$, if there are $u \in[x], v \in[y]$ such that $u \preceq v$, we write $[x] \preceq[y]$.

Lemma 8 (see [11]). Let $E$ be an incomplete preference set, and $\Omega$ is a subset in $E$. The order relation $\leq$ in quotient set $\Omega / \sim$ which is elicited by the incomplete preference relation $\preceq$ in $E$ is a partial order. Then the quotient set $\Omega / \sim$ is a poset.

Lemma 9 (see [11]). If $\Omega$ is incomplete preference pseudo separable, then $\Omega / \sim$ is incomplete preference pseudo separable.
Lemma 10 (see [12] (Zorn Lemma)). Let E be a nonempty partial ordered set. If every total ordered subset in $E$ has an upper bound in $E$, then there is a maximal element in $E$.

\section{Existence Theorems for Fixed Point on Incomplete Preference Sets}

Partial order method is discussed and applied greatly in mathematics, and the conclusion on the partial order is becoming a very complete system [13-23]. But few scholars study the fixed point and extreme value theorems on incomplete preference set.

Definition 11. Let $\left(E, \preceq^{E}\right),\left(U, \preceq^{U}\right)$ be incomplete preference sets, and let $T: E \longrightarrow 2^{U}$ be an order-increasing set-valued mapping. $T$ is said to be order-increasing upward, if $x \preceq^{E} y$ in $E$, for any $u \in T(x)$; there is $v \in T(y)$ such that $u \unlhd^{U} v$; $T$ is said to be order-increasing downward, if $x \unlhd^{E} y$ in $E$, for any $v \in T(y)$; there is $u \in T(x)$ such that $u \preceq{ }^{U} v$. If $T$ is both order-increasing upward and order-increasing downward, $T$ is said to be order-increasing.

Definition 12. Let $(E, \preceq)$ be incomplete preference set, and let $T: E \longrightarrow 2^{E} \backslash \Phi$ be an order-increasing set-valued mapping. An element $x \in E$ is called a generalized fixed point of $T$, if there are $x^{*} \in E, u \in T x^{*}$ such that $x^{*} \sim u$.

Let $(E, \preceq)$ be incomplete preference set, and let $T: E \longrightarrow$ $2^{u}$ be an order-increasing set-valued mapping. The following notation will be used in Theorem 13:

$$
S T(x)=\{x \in E \mid x \preceq u, y \in T(x)\} .
$$

Theorem 13. Let $(E, \preceq)$ be an incomplete preference pseudo separable set, and let $T: E \longrightarrow 2^{E}$ be an order-increasing setvalued mapping. If $T$ satisfies the following conditions:

$\left(A_{1}\right)$ Every increasing sequence in $S T(x)$ has an upper bound in $S T(x)$

$\left(A_{2}\right)$ There is a $x_{0} \in E$ with $x_{0} \preceq u$, for some $u \in T x_{0}$

then $T$ has a generalized fixed point; that is, there are $x^{*} \in$ $E, u \in T x^{*}$ such that $x^{*} \sim u$.

Proof. Let $\Omega=\{x \in E \mid x \preceq u, y \in T(x)\}$. From the condition $\left(A_{2}\right)$, it implies that $\Omega$ is a nonempty set in $E$. Take an arbitrary total ordered subset $M \subset \Omega$. Since $M$ is also an arbitrary total ordered subset of incomplete preference pseudo separable set $(E, \preceq)$, there is denumerable set $\left\{x_{n}\right\} \subset$ $M$ such that if $x \in M, x \neq \sup M$, there is $x_{n_{0}} \in\left\{x_{n}\right\}$ which satisfy $x \preceq x_{n_{0}}$.

Let

$$
\begin{aligned}
& z_{1}=x_{1}, \\
& z_{n}=\max \left\{x_{n}, z_{n-1}\right\}, \quad n=2,3 \cdots . \\
& z_{n} \subset M \subset \Omega(x) .
\end{aligned}
$$

Since $M$ is an arbitrary total ordered subset, $\left\{z_{n}\right\}$ is well defined. So

$$
z_{1} \preceq z_{2} \preceq \cdots z_{n} \preceq \cdots
$$


For any $x \in M, x \neq \sup M$, by the condition $\left(A_{1}\right)$, there is a point $z^{*} \in \Omega$ such that $z_{n} \preceq z^{*}$, and since $(E, \preceq)$ is an incomplete preference pseudo separable set, there is a $x_{n_{0}} \in\left\{x_{n}\right\}$ such that $x \leq x_{n_{0}}$. By the definition of $\left\{z_{n}\right\}$, we get

$$
x \preceq x_{n_{0}} \preceq z_{n} \preceq z^{*} .
$$

That is, $z^{*} \in \Omega$ is an upper bound of total ordered subset $M$.

Let $[x]=\{y \in \Omega \mid x \sim y\}$; then $[x]$ is equivalence class set in $\Omega$. Assuming that $\Omega / \sim=\{[x], x \in \Omega\}$ is a quotient set corresponding to the equivalence relation $\sim$, then applying Lemmas 8 and 9 , we get that the order relation $\leq$ in quotient set $\Omega / \sim$ which is elicited by the incomplete preference relation $\preceq$ in $\Omega$ is a partial order.

Take an arbitrary total ordered subset $N \subset \Omega / \sim$, next, to show that the set $N$ has an upper bound in $\Omega / \sim$.

Let

$$
W=\bigcup[x] \subset \Omega .
$$

It is easy to know that $W$ is total ordered subset in $\Omega$. In fact, for any $x, y \in W$, there are $[x],[y] \in N$. Since $N$ is total ordered subset in $\Omega / \sim$, we can get that either $[x] \leq[y]$ or $[y] \leq[x]$ is valid. According to the relation between partial order and incomplete preference, we have that either $x \leq y$ or $y \leq x$ is valid. So $W$ is total ordered subset in $\Omega$.

For any $[z] \in N$, by the definition of $W$, we get $z \in W$. Since every total ordered subset in $\Omega$ has an upper bound, there is $x_{0} \in W$ such that $z \leq x_{0}$. So $[z] \leq\left[x_{0}\right]$; that is, every total ordered subset in $\Omega / \sim$ has an upper bound. Then applying Zorn lemma, we have that there is a maximal element $\left[x^{*}\right]$ in $\Omega / \sim$. By the definition of $\Omega / \sim$, we have that $x^{*}$ is the maximal element in $\Omega$.

Since $x^{*}$ is the maximal element in $\Omega$, there is $u \in T\left(x^{*}\right)$ such that $x^{*} \preceq u$. Supposing that $u \npreceq x^{*}$, the monotonicity of $T$ together with $u \in T\left(x^{*}\right), x^{*} \preceq u$ implies that there is $v \in$ $T(u)$ such that $u \preceq v$. It means $u \in \Omega$, and it is contradictory with which $x^{*}$ is the maximal element in $\Omega$. So $u \preceq x^{*}$ is proved. Hence there are $x^{*} \in E, u \in T x^{*}$ such that $x^{*} \sim u$; that is, $T$ has a generalized fixed point.

Corollary 14. Let $(E, \preceq)$ be an incomplete preference pseudo separable set, and let $T: E \longrightarrow 2^{E} \backslash \Phi$ be an order-increasing set-valued mapping. If $T$ satisfies the following conditions:

$\left(A_{1}\right)$ Every increasing sequence in $S T(x)$ has an upper bound in $S T(x)$

$\left(A_{2}\right)$ There is $x_{0} \in E$ with $x_{0} \preceq u$, for some $u \in T x_{0}$

$\left(A_{3}\right)$ If $x \sim y, x, y \in E$, then $T x=T y$

then $T$ has a fixed point; that is, there are $x^{*} \in E, u \in T x^{*}$ such that $x^{*} \in T x^{*}$.

\section{Existence of Nash Equilibrium Points in Generalized Games under Incomplete Preferences}

The incomplete preference we present in the paper is more general order relation than the preference in the field of economic management and coincident with the reality of economic phenomenon. It can be applied to the existence of generalized Nash equilibrium of noncooperative game theory.

Definition 15. Let $n$ be a positive integer greater than 1 . An $n$-person noncooperative game consists of the following elements:

(1) The set of $n$ players is denoted by $I=\{i=1,2,3, \ldots, n\}$;

(2) For any $i \in I$, let $S_{i}$ be the strategy set of player $i$ and $\left(S_{i}, \preceq_{i}\right)$ be an incomplete preference pseudo separable set; denote $S=S_{1} \times S_{2} \times \cdots \times S_{n}$;

(3) Let $P_{i}: S \longrightarrow U, i=1,2,3, \ldots, n$ be the payoff function for player $i$; denote $P=\left\{P_{1}, P_{2}, \ldots, P_{n}\right\}$.

The game is denoted by $\Gamma=(N, S, P, U)$.

Every player in the $n$-person noncooperative game independently chooses his own strategy $x_{i} \in S_{i}, i=1,2,3, \ldots, n$, to maximize his payoff function $P_{i}\left(x_{1}, x_{2}, \ldots, x_{n}\right) \in U$. For any $x=\left(x_{1}, x_{2}, \ldots, x_{n}\right) \in S$, denote

$$
\begin{aligned}
& x_{-i}=\left(x_{1}, x_{2}, \ldots, x_{i-1}, x_{i+1}, \ldots, x_{n}\right) \in S, \\
& S_{-i}=S_{1} \times S_{2} \times \cdots \times S_{i-1} \times S_{i+1} \times \cdots \times S_{n} .
\end{aligned}
$$

Then $x_{-i} \in S_{-i}$, and $x$ can be written as $x=\left(x_{i}, x_{-i}\right)$.

Definition 16. Let $\Gamma=(N, S, P, U)$ be an $n$-person noncooperative game. The strategy $\widehat{x}=\left(\widehat{x}_{1}, \widehat{x}_{2}, \ldots, \widehat{x}_{n}\right) \in S$ is said to be a generalized Nash equilibrium in the noncooperative game $\Gamma=(N, S, P, U)$ under the incomplete preference, if there is strategy $\widehat{x}=\left(\widehat{x}_{1}, \widehat{x}_{2}, \ldots, \widehat{x}_{n}\right) \in S$, for every $i=1,2,3, \ldots, n$; the following order inequality holds

$$
P_{i}\left(x_{i}, \widehat{x}_{-i}\right) \nsucceq^{U} P_{i}\left(\widehat{x}_{i}, \widehat{x}_{-i}\right), \quad \forall x_{i} \in S_{i} .
$$

Lemma 17. Let $\left(S_{i}, \preceq^{s_{i}}\right)$ be an incomplete preference pseudo separable set. $S=S_{1} \times S_{2} \times \cdots \times S_{n}$ is a coordinate ordering set composed of $S_{1}, S_{2}, \ldots, S_{n}$, for any $x=\left(x_{1}, x_{2}, \ldots, x_{n}\right), y=$ $\left(y_{1}, y_{2}, \ldots, y_{n}\right) \in S$, the order relation $\unlhd^{s}$ in $S$ induced by the partial order $\leq^{S_{i}}$, denoted as the following:

$$
x \preceq^{s} y \Longleftrightarrow x_{i} \preceq^{S_{i}} y_{i}, \quad \forall i=1,2,3, \ldots, n .
$$

Then $\left(S, \preceq^{s}\right)$ is an incomplete preference pseudo separable set.

Proof. First we show that $\left(S, \leq^{S}\right)$ is an incomplete preference set. Since $\left(S_{i}, \preceq^{s_{i}}\right)$ is incomplete preference set, for any $x=$ $\left(x_{1}, x_{2}, \ldots, x_{n}\right) \in S$, we can get $x_{i} \preceq^{s_{i}} x_{i}, \forall i=1,2,3, \ldots, n$. So $x \preceq^{s} x$. Hence the order relation $\unlhd^{s}$ satisfies reflexive axiom.

For any $x=\left(x_{1}, x_{2}, \ldots, x_{n}\right), y=\left(y_{1}, y_{2}, \ldots, y_{n}\right)$, and $z=\left(z_{1}, z_{2}, \ldots, z_{n}\right) \in S$, which satisfy $x \leq^{s} y, y \preceq^{s} z \in S$. By Definition 15, we have $x_{i} \leq^{S_{i}} y_{i}, y_{i} \leq^{S_{i}} z_{i}$. Since $\left(S_{i}, \leq^{S_{i}}\right)$ is incomplete preference set, we have $x_{i} \leq^{s_{i}} z_{i}$. So $x \leq^{s} z$; that is, $S=S_{1} \times S_{2} \times \cdots \times S_{n}$ is incomplete preference set.

Next we prove that the incomplete preference set $\left(S, \varliminf^{s}\right)$ is pseudo separable, for any $x=\left(x_{1}, x_{2}, \ldots, x_{n}\right) \in S$, since $\left(S_{i}, \preceq^{s_{i}}\right)$ is pseudo separable,

Let $M_{i}$ be an arbitrary total ordered subset of set $S_{i}$; then there is denumerable set $\left\{x_{i}^{n}\right\} \subset M_{i}$ such that if $x_{i} \in M_{i}, x_{i} \neq$ $\sup M_{i}$, there is $x_{i_{n_{0}}}^{n} \in\left\{x_{i}^{n}\right\}$ which satisfies $x_{i}^{n} \leq x_{i_{n_{0}}}^{n}$.

Define the following

$$
M=M_{1} \times M_{2} \times \cdots \times M_{n} .
$$


Let $x^{n}=\left\{x_{1}^{n}, x_{2}^{n}, \ldots, x_{n}^{n}\right\}, x^{n}{ }_{n_{0}}=\left\{x_{1 n_{0}}^{n}, x_{2 n_{0}}^{n}, \ldots, x_{n n_{0}}^{n}\right\}$; then by the definition of $M$, we have $x^{n} \in M$. This together with $x^{n}{ }_{n_{0}} \in\left\{x^{n}\right\}$ implies that $x^{n} \preceq^{s} x^{n}{ }_{n_{0}}$. Hence the incomplete preference set $\left(S, \preceq^{s}\right)$ is pseudo separable.

Theorem 18. Let $\Gamma=(N, S, P, U)$ be an $n$-person noncooperative game. Suppose that, for any $x \in S$, the payoff function $P_{i}, i=1,2,3, \ldots, n$, satisfies the following conditions:

$\left(G_{1}\right)$ Every total ordered subset in $P_{i}\left(S_{i}, x_{-i}\right)$ has an upper bound in $P_{i}\left(S_{i}, x_{-i}\right)$;

$\left(G_{2}\right)$ Every increasing sequence in the inverse image $\left\{z_{i} \in\right.$ $S_{i}: P_{i}\left(S_{i}, x_{-i}\right)$ is a maximal element of $\left.P_{i}\left(S_{i}, x_{-i}\right)\right\}$ has an upper bound;

$\left(G_{3}\right)$ For any $x, y \in S, x \preceq^{S} y$, if there is $z_{i} \in S_{i}$ with $P_{i}\left(z_{i}, x_{-i}\right)$ to be a maximal element of $P_{i}\left(S_{i}, x_{-i}\right)$, then there is $\omega_{i} \in S_{i}$ with $z_{i} \preceq^{s_{i}} \omega_{i}$ such that $P_{i}\left(\omega_{i}, y_{-i}\right)$ is a maximal element of $P_{i}\left(S_{i}, y_{-i}\right)$;

$\left(G_{4}\right)$ If there are $p, q \in S$ such that $p \preceq^{s} q$ and $P_{i}\left(q_{i}, p_{-i}\right)$ is a maximal element of $P_{i}\left(S_{i}, p_{-i}\right)$;

$\left(G_{5}\right)$ If $p \sim^{s} q, p, q \in S$ such that $P_{i}\left(p_{i}, x_{-i}\right)=P_{i}\left(q_{i}, x_{-i}\right)$.

Then there is a generalized Nash equilibrium in the $n$-person noncooperative game $\Gamma=(N, S, P, U)$.

Proof. Since $\left(S_{i}, \preceq^{s_{i}}\right)$ is an incomplete preference pseudo separable set, for every $i=1,2,3, \cdots, n$, then, from Lemma 17, $\left(S, \preceq^{s}\right)$ is also an incomplete preference pseudo separable set equipped with the product order $\preceq^{s}$.

For every fixed $i=1,2,3, \ldots, n$, define a set-valued mapping $T_{i}: S \longrightarrow 2^{S_{i}} \backslash \Phi$ as the following:

$$
\begin{aligned}
& T_{i}(x)=\left\{z_{i} \in S_{i}:\right. \\
& \left.\quad P_{i}\left(S_{i}, x_{-i}\right) \text { is a maximal element of } P_{i}\left(S_{i}, x_{-i}\right)\right\},
\end{aligned}
$$

for all $x=\left(x_{1}, x_{2}, \ldots, x_{n}\right) \in S$.

From assumption $G_{1}$ of this theorem, for every fixed element $x \in S$, every total ordered subset in $P_{i}\left(S_{i}, x_{-i}\right)$ has an upper bound in $\left(U, \preceq^{U}\right)$. Then applying Zorn Lemma, the set $\left.P_{i}\left(S_{i}, x_{-i}\right)\right\}$ has a maximal element. Therefore, $T_{i}(x)$ is a nonempty subset of $S_{i}$. Then we define

$$
T(x)=T_{1}(x) \times T_{2}(x) \times \cdots \times T_{n}(x), \quad x \in S .
$$

For any arbitrary $x \in S$, with respect to the set $T(x)$, we write

$$
S T(x)=\left\{x \in S: x \preceq^{s} z, z \in T(x)\right\} .
$$

For every $i=1,2,3, \ldots, n$, we have

$$
\begin{aligned}
& S T_{i}(x)=\left\{x_{i} \in S_{i}: x_{i} \preceq^{s_{i}} z_{i}, z_{i} \in T_{i}(x)\right\}=\left\{x_{i} \in S_{i}: x_{i}\right. \\
& \quad \varsigma^{s_{i}} z_{i}, z_{i} \in S_{i}, \\
& \left.\quad P_{i}\left(z_{i}, x_{-i}\right) \text { is a maximal element of } P_{i}\left(S_{i}, x_{-i}\right)\right\} .
\end{aligned}
$$

Now we prove that the operator $T$ satisfies the conditions in Theorem 18. Firstly, we will show that the operator $T$ is order-increasing. For any given $x \preceq^{S} y$ in $S$, and for any $z=$ $\left(z_{1}, z_{2}, \ldots, z_{n}\right) \in T(x)$, for every $i=1,2, \ldots, n$, we have $z_{i} \in T_{i}(x)$; that is, $P_{i}\left(z_{i}, x_{-i}\right)$ is a maximal element of $P_{i}\left(S_{i}, x_{-i}\right)$. Then from hypothesis $G_{3}$ of this theorem, there is $\omega_{i} \in S_{i}$ with $z_{i} \preceq^{s_{i}} \omega_{i}$ such that $P_{i}\left(\omega_{i}, y_{-i}\right)$ is a maximal element of $P_{i}\left(S_{i}, y_{-i}\right)$; that is, $\omega_{i} \in T_{i}(y)$. Let $\omega=\left(\omega_{1}, \omega_{2}, \ldots, \omega_{n}\right)$. We obtain that $z \preceq^{S} \omega$ and $\omega \in D(y)$. Hence the operator $T$ is order-increasing.

From assumption $G_{2}$ of this theorem, every increasing sequence in $T_{i}(x)$ has an upper bound. Then we can similarly show that every increasing sequence in $S T_{i}(x)$ has an upper bound. In fact, take an arbitrary total ordered subset $M \subset$ $S T(x)$. Since $M$ is also a total ordered subset of incomplete preference pseudo separable set $(E, \succeq)$, there is denumerable set $\left\{x_{n}\right\} \subset M$ such that for any $x \in M, x \neq \sup M$, there are $x_{n_{0}} \in\left\{x_{n}\right\}, u(x) \in T(x)$, which satisfy $x \preceq^{S} x_{n_{0}}, x \preceq^{S} u(x)$.

From the above discussion, we have that there is $e(x) \in$ $T\left(x_{n_{0}}\right)$ such that $u(x) \preceq^{S} e(x)$. Thus we obtain a mapping $e$ : $S T(x) \longrightarrow T(x)$ satisfying the following order inequality: $x \preceq u(x) \preceq^{S} e(x), u(x) \in T(x)$ with $e(x) \in T(x)$. For any an increasing sequence $\left\{x_{n}\right\}$ in $S T(x)$, we have that there is $z^{*} \in \Omega$ such that $x_{n} \preceq^{S} z^{*}$. By the definition of $\left\{z_{n}\right\}$, we get

$$
x \preceq^{S} x_{n_{0}} .
$$

So we have that $\left\{x_{n}\right\}$ is an increasing sequence; then $\left\{e\left(x_{n}\right)\right\}$ is an increasing sequence in $T(x)$. since every increasing sequence in $T(x)$ has an upper bound in $T(x)$, we have that there is $z^{*} \in T(x)$ such that $e\left(x_{n}\right) \preceq^{S} z^{*}$. Since $x_{n} \preceq^{S} e\left(x_{n}\right)$, we have $x_{n} \preceq^{S} z^{*}$. Hence every increasing sequence in $S T(x)$ has an upper bound in $S T(x)$.

Then applying Theorem 13, it implies that the operator $T$ has a generalized point; that is, there are $x^{*} \in E, u \in T x^{*}$ such that $x^{*} \sim^{S} u$. Since the operator $T$ is order-increasing, there are $u \in T\left(x^{*}\right)$ and $v \in T(u)$ such that $u \varsigma^{s} v$.

From assumption $G_{5}$ of this theorem, if $x^{*} \sim^{S} u$, we can get

$$
P_{i}\left(x_{i}^{*}, x_{-i}\right)=P_{i}\left(u_{i}, x_{-i}\right) .
$$

Since $u \in T\left(x^{*}\right)$, we have that $P_{i}\left(u_{i}, x_{-i}^{*}\right)$ is a maximal element of $P_{i}\left(S_{i}, x_{-i}^{*}\right)$. This together with $P_{i}\left(x_{i}^{*}, x_{-i}\right)=$ $P_{i}\left(u_{i}, x_{-i}\right)$ implies that $P_{i}\left(x_{i}^{*}, x_{-i}^{*}\right)$ is a maximal element of $P_{i}\left(S_{i}, x_{-i}^{*}\right)$. It implies that for any $x^{*}=\left(x_{1}^{*}, x_{2}^{*}, \ldots, x_{n}^{*}\right) \in S$, $i=1,2,3, \ldots, n$, the following inequality is established

$$
P_{i}\left(x_{i}, x_{-i}^{*}\right) \nsucceq^{U} P_{i}\left(x_{i}^{*}, x_{-i}\right), \quad \forall x_{i} \in S_{i} .
$$

This shows that $x^{*}=\left(x_{1}^{*}, x_{2}^{*}, \ldots, x_{n}^{*}\right) \in S$ is a generalized Nash equilibrium in the $n$-person noncooperative game $\Gamma=$ $(N, S, P, U)$.

\section{Conclusion}

Incomplete preference is more general order relation than complete preference in the field of economic management, because restriction on order relation is eased. So it is more consistent with the reality of economic management phenomenons. The generalized game model under the incomplete preference can play an important application in economic management problems. Generalized game plays an important role to prove existence of general equilibrium. 
But many economic problems ultimately come down to nonlinear problems which are denoted by the utility function without preference in an order infinite dimensional space. The traditional general game model cannot deal with the problems such as the utility function without preference, incomplete preference, order infinite dimension space, or nonlinear problem. Now since there are no ready-made methods to deal with the problems, new research methods must be sought. The generalized game model under the incomplete preference, which is proposed in this paper, can deal with the problems. But in this paper, the research is limited to the existence of the equilibrium. The stability of equilibrium and new game model which are more close to the reality are our next step research direction.

\section{Data Availability}

No data were used to support this study.

\section{Conflicts of Interest}

The author declares that there are no conflicts of interest regarding the publication of this article.

\section{Acknowledgments}

The author was supported by the National Social Science Fund of China (13CJL006), National Science Foundation for Post-doctoral Scientists of China (2014M551264), and Research Fund for the Doctoral Program of Changshu Institute of Technology (XE1509).

\section{References}

[1] M. Wu, L. Li, and Z. Sun, "Transposable element fragments in protein-coding regions and their contributions to human functional proteins," Gene, vol. 401, no. 1-2, pp. 165-171, 2007.

[2] J.-C. Hou, "Existence of equilibria for generalized games without paracompactness," Nonlinear Analysis. Theory, Methods \& Applications. An International Multidisciplinary Journal, vol. 56, no. 4, pp. 625-632, 2004.

[3] J. V. Llinares, "Existence of equilibrium in generalized games with abstract convexity structure," Journal of Optimization Theory and Applications, vol. 105, no. 1, pp. 149-160, 2000.

[4] J.-C. Hou, "An existence theorem of equilibrium for generalized games in $H$-spaces," Applied Mathematics Letters, vol. 16, no. 1, pp. 97-103, 2003.

[5] J. von Neumann and O. Morgenstern, Theory of Games and Economic Behavior, Princeton, NJ, USA, Princeton University Press, 2nd edition, 1947.

[6] R. J. Aumann, "Utility Theory without the Completeness Axiom," Econometrica, vol. 30, no. 3, p. 445, 1962.

[7] B. Truman, "Knightian decision theory. Part I," Cowles Foundation Discussion, 1986.

[8] D. Schmeidler, "Competitive equilibria in markets with a continuum of traders and incomplete preferences," Econometrica, vol. 37, pp. 578-585, 1969.

[9] Da. Junguo, Nonlinear Functional Analysis, Shandong Science and Technology Press, Jinan, 2001.
[10] A. Ok Efe, P. Ortoleva, and G. Riella, "Incomplete Preferences under Uncertainty: Indecisiveness in Beliefsvs Tastaes," Econometrica: Journal of the Econometric Society, vol. 80, no. 4, pp. 1791-1808, 2012.

[11] Zhang. Jinqin, Ordering Method and Equilibrium Analysis[M]., Shanghai, Fudan University publishing house, 2003.

[12] J. Xiong, The Handout of Point Set Topology, The Peoples Education Press, Beijing, 1981.

[13] Z. Zhao, "Positive solutions of semi-positone Hammerstein integral equations and applications," Applied Mathematics and Computation, vol. 219, no. 5, pp. 2789-2797, 2012.

[14] K. M. Zhang, "On a sign-changing solution for some fractional differential equations," Boundary Value Problems, vol. 2017, no. 59,8 pages, 2017.

[15] Xinsheng Du and Anmin Mao, "Existence and Multiplicity of Nontrivial Solutions for a Class of Semilinear Fractional Schrödinger Equations," Journal of Function Spaces, vol. 2017, Article ID 3793872, 7 pages, 2017.

[16] J. Liu and A. Qian, "Ground state solution for a SchrödingerPoisson equation with critical growth," Nonlinear Analysis: Real World Applications, vol. 40, pp. 428-443, 2018.

[17] X. Zhang, L. Liu, Y. Wu, and B. Wiwatanapataphee, "Nontrivial solutions for a fractional advection dispersion equation in anomalous diffusion," Applied Mathematics Letters, vol. 66, pp. $1-8,2017$.

[18] D. Kong, L. Liu, and Y. Wu, "Isotonicity of the metric projection by Lorentz cone and variational inequalities," Journal of Optimization Theory and Applications, vol. 173, no. 1, pp. 117-130, 2017.

[19] J. Mao, Z. Zhao, and N. Xu, "The existence and uniqueness of positive solutions for integral boundary value problems," Bulletin of the Malaysian Mathematical Sciences Society, vol. 34, no. 1, pp. 153-164, 2011.

[20] J. Mao, Z. Zhao, and N. Xu, "On existence and uniqueness of positive solutions for integral boundary value problems," Electronic Journal of Qualitative Theory of Differential Equations, vol. 16, pp. 1-8, 2010.

[21] Y. Guan, Z. Zhao, and X. Lin, "On the existence of positive solutions and negative solutions of singular fractional differential equations via global bifurcation techniques," Boundary Value Problems, vol. 2016, no. 1, article 141, 2016.

[22] Z. Zhao, "Existence and uniqueness of fixed points for some mixed monotone operators," Nonlinear Analysis: Theory, Methods \& Applications, vol. 73, no. 6, pp. 1481-1490, 2010.

[23] J. J. Nieto and R. Rodríguez-López, "Existence and uniqueness of fixed point in partially ordered sets and applications to ordinary differential equations," Acta Mathematica Sinica, vol. 23, no. 12, pp. 2205-2212, 2007. 


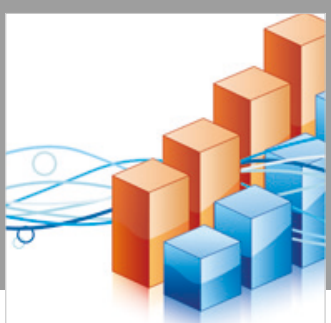

Advances in

Operations Research

\section{-n-m}
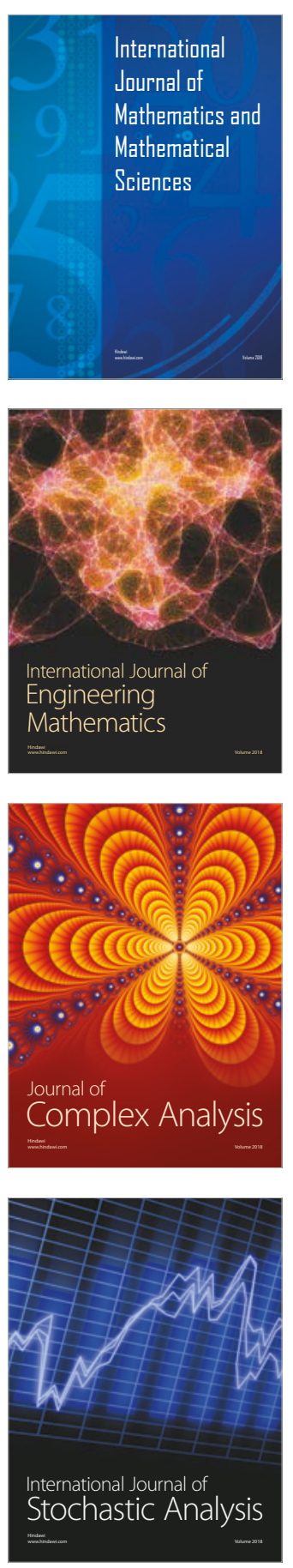
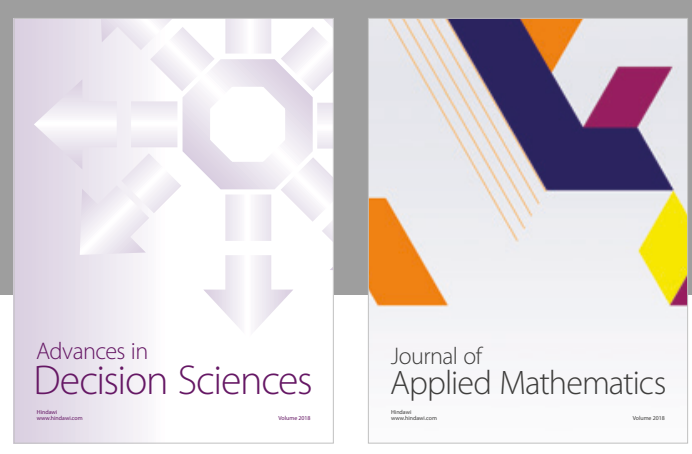

Journal of

Applied Mathematics
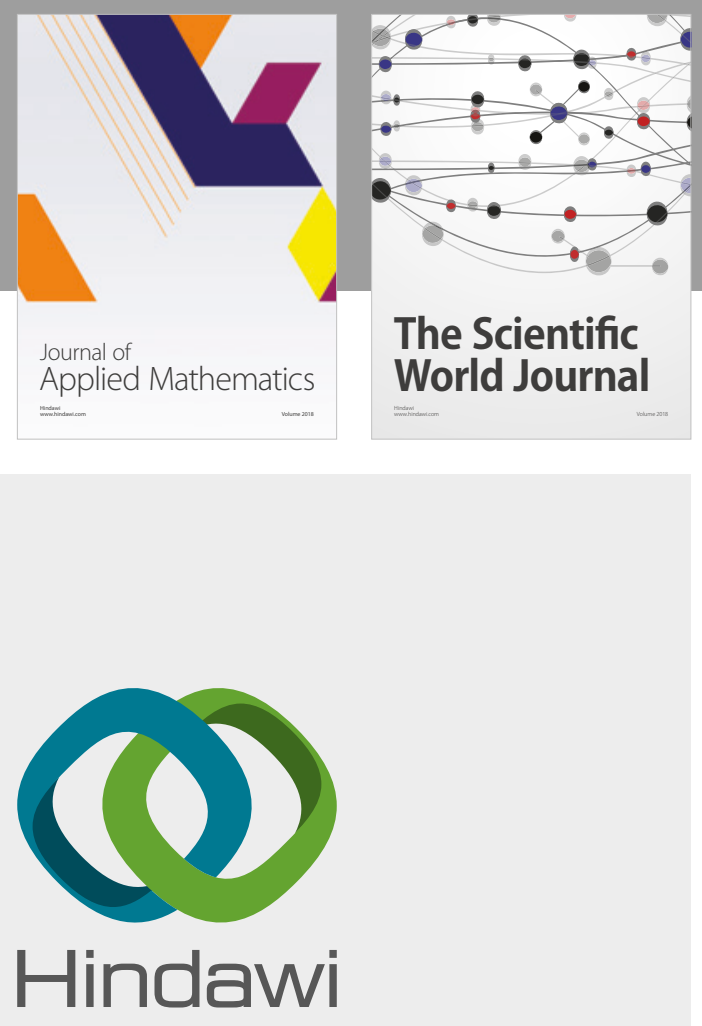

Submit your manuscripts at

www.hindawi.com

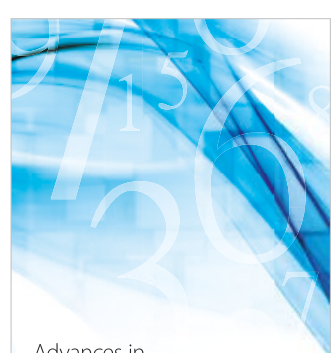

Advances in
Numerical Analysis
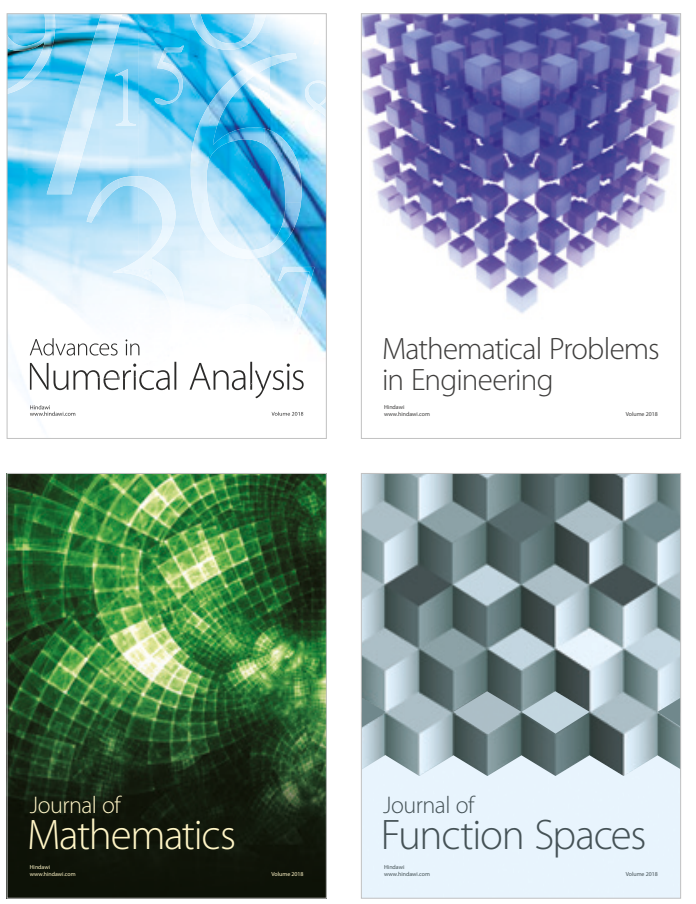

Mathematical Problems in Engineering

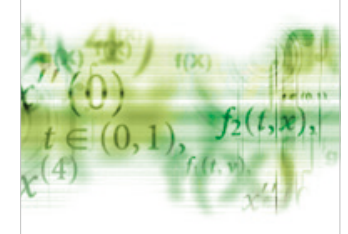

International Journal of

Differential Equations

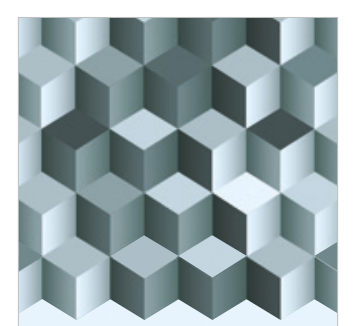

Journal of

Function Spaces

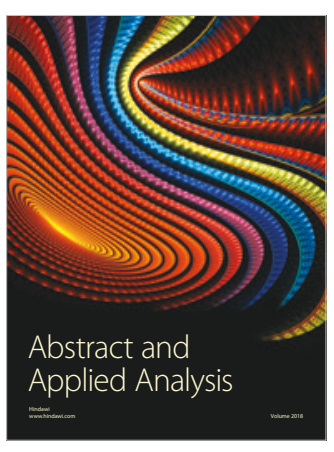

The Scientific

World Journal

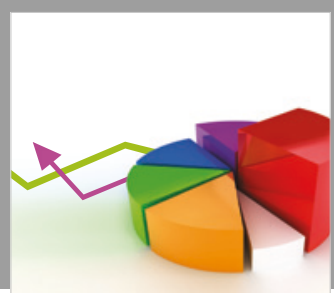

Journal of

Probability and Statistics
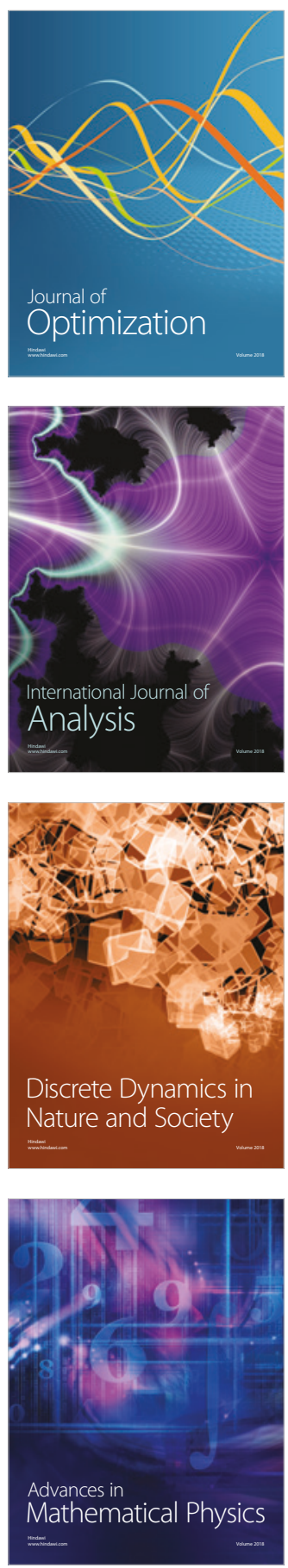